\title{
Nota sobre culicídeos (Diptera: Culicidae) da bacia do rio Purus, Acre, Amazônia (Brasil)
}

\author{
Note on the culicidae (Diptera: Culicidae) of the River Purus Basin, Acre, \\ Amazonian, Brazil
}

\author{
Delsio Natal*, José Maria Soares Barata*, Carmen Beatriz Taipe Lagos*, Roraima Moreira Rocha** \\ NATAL, D. et al. Nota sobre culicídeos (Diptera: Culicidac) da Bacia do Rio Purus, Acre, Amazônia \\ (Brasil) - Rev. Saúde públ., S. Paulo, 26: 129-31, 1992. Foram fcitas coletas de mosquito (Diptera: \\ Culicidae) na área do projeto de Colonização Pedro Peixoto, no Estado do Acre, Brasil. Obteve-se um \\ total de 4.588 exemplares pertencentes a 53 espécies ou grupos. Salienta-se a ocorrência de \\ Anopheles (Nyssorhynchus) oswaldoi.
}

Descritores: Mosquitos. Ecologia de vetores.

De maneira semclhante a outras áreas da Rcgiāo Amazônica, o Estado do Acre vem sofrendo cada vez mais as conscqüências decorrentes do processo de colonização.

A abertura de rodovias tem possibilitado a ampliação da fronteira agrícola à medida que o desmatamento progride e novas árcas são ocupadas por famílias migrantes.

No ambiente desmatado, onde se instalam os trabalhadores, formam-se condições propícias ao desenvolvimento de culicídeos que são incriminados como vetores de doenças ao homem.

É esparso o conhecimento da fauna de culicídeos da Amazônia. Em geral, a maior preocupação dos autores tem se restringido ao estudo dos anofelinos dada a importância que representam na transmissão da malária (Deane e col${ }^{4}, 1946$; Arruda e col$^{2}, 1986$; Tadci e col ${ }^{14}, 1983$ ).

A presente nota tem como objetivo comunicar a identificação de culicídcos adultos colctados $\mathrm{cm}$ um projeto de assentamento agrícola, como contribuição ao conhecimento da fauna local.

A preocupação com o cstudo da launa nessa árca de desmatamento recente é de extrema importância, pois espera-se a curto prazo uma profunda modificação na composição das espécics como

\footnotetext{
* Departamento de Epidemiologia da Faculdade de Saúde Pública da Universidade de São Paulo - São Paulo, SP . Brasil

** Fundação Nacional de Saúde (PNS) - Rio Branco, Acre - Brasil
}

Separatas/Reprints: D. Natal - Av. Dr. Amaldo, 715 - 01246. 904 - São Paulo, SP - B rasil

Publicação financiada pela FAPESP. Processo Saúdc Colctiva 91/4994.0 conscqüência da alteração ambiental.

Foi escolhida para as coletas a região ocupada pelo Projeto de Colonização Pedro Peixoto, na Bacia do Rio Purus, que se localiza nos municípios de Rio Branco, Scnador Guiomard e Plácido de Castro, entre as latitudes de $9^{\circ} 06^{\prime} \mathrm{e} 10^{\circ} 30^{\prime} \mathrm{S}$ e as longiludes de $67^{\circ} 00^{\prime}$ e $67^{\circ} 40^{\prime} \mathrm{W}$.

Os culicídcos foram coletados nos meses de agosto, selembro e oulubro de 1990 e janeiro de 1991. Foram fcitas 44 coletas $\mathrm{cm}$ diferentes pontos da região. As coletas foram realizadas durante 0 crepúsculo vespertino e início da noite (até 21:00 h), no peridomicílio $\mathrm{c}$ áreas abertas do meio rural. As técnicas de colcta foram: isca humana, armadilha de Shannon, aspirador elétrico e coleta direta em isca animal (gado bovino). As coletas não foram padronizadas, visando a comparaçōes de freqücencias nos diferentes habitats ou nos diferentes horários. A razão do cmprego das variadas técnicas de coleta foi com a intenção de se obler o maior número possivel de espécics. Um estudo mais aprofundado com vistas a avaliação da atividade culicidiana não foi possivel, pois as coletas foram realizadas acompanhando-sc o deslocamento de rotina de uma equipe da Fundação Nacional de Saúde (FNS).

A idenulficação procedeu-se com auxílio bibliográlico (Lane ${ }^{10}$, 1953; Galindo e col. ${ }^{8}$, 1954; Forattini $^{7}, 1962 / 1965$; Gucdes e Miguel $^{9}, 1964$; Arnell 1 , 1976; Faran 5 , 1980; Faran e Linthicum ${ }^{6}$, 1981; Sirivanakarns ${ }^{13}$, 1982; Linthicum ${ }^{11}$, 1988; Rosa-Frcilas ${ }^{12}, 1989$ ). A confirmação da identificação foi feita por meio de comparação com material da coleção entomológica da Faculdade de Saúde Pública da USP. Após cssa verificação, excmplares representando a fauna identificada foram depositados na referida coleção. 
Tabela. Distribuição de culicideos adultos identificados na Bacia do Rio Purus, Estado do Acre, em coletas realizadas em agosto, setembro e outubro de 1990 e janeiro de 1991.

\begin{tabular}{|c|c|c|}
\hline Identificação & Quantidade & $\%$ \\
\hline $\begin{array}{l}\text { Anopheles (Nyssorhynchus) oswaldoi } \\
\text { Anopheles (Nyssorhynchus) } \\
\text { deaneorum }\end{array}$ & $\begin{array}{r}3.156 \\
378\end{array}$ & $\begin{array}{r}68,8 \\
8,2\end{array}$ \\
\hline Aedes (Ochlerotatus) scapularis & 296 & 6,4 \\
\hline $\begin{array}{l}\text { Culex (Melanoconion) spp. } \\
\text { Anopheles (Nyssorhynchus) }\end{array}$ & $\begin{array}{r}134 \\
94\end{array}$ & $\begin{array}{l}2,9 \\
2,0\end{array}$ \\
\hline triannulatus & & \\
\hline $\begin{array}{l}\text { Culex (Culex) sp. grupo coronator } \\
\text { Coquillettidia (Rhynchotaenia) }\end{array}$ & $\begin{array}{l}77 \\
64\end{array}$ & $\begin{array}{l}1,7 \\
1,4\end{array}$ \\
\hline venezuelensis & & \\
\hline $\begin{array}{l}\text { Mansonia (Mansonia) amazonensis } \\
\text { Anopholes (Nyssorhynchus) darlingi }\end{array}$ & 53 & $\begin{array}{l}1,2 \\
1,0 \\
1,0\end{array}$ \\
\hline Outras espécies (com \% < que 1\%)" & 290 & 6,4 \\
\hline & & 100,0 \\
\hline
\end{tabular}

- (ordem decrescente de quantidade): Mansonia (Mansonia) sp., Mansonia (Mansonia) humeralis, Culex (Culex) spp., Psorophora (Grabhamia) cingulata, Coquillettidia (Rhynchotaenia) juxtamansonia, Culex (Culex) sp. pr. declarator, Coquillettidia (Rhynchotaenia) nigricans, Culex (Melanoconion) sp. gr. pilosus, Coquillettidia (Rhynchotaenia) albicosta, Coquillettidia (Rhynchotaenia) sp., Culex (Melanoconion) dunni, Culex (Melanoconion) inadmirabilis, Culex (Melanoconion) bequaerti, Aedomyia (Aedomyia) squamipennis, Anopheles (Nyssorhinchus) strodei, Psorophora (Psorophora) cillata, Anopheles (Nyssorhynchus) nuneztovari, Culex (Melanoconion) theobaldi, Psorophora (Janthinosoma) sp. pr. Albigenu, Aedes (Ochle. rotatus) serratus, Culex (Culex) bibens, Culex (Melonoconion) bastagarius, Culex (Melanoconion) sp. gr. atratus, Uranotaenia (Uranotaenia) pulcherrima, Psorophora (Janthinosoma) sp., Culex (Melanoconion) sp. gr. inhibitator, Anopheles (Nyssorhynchus) galvaioi, Anopheles (Anopheles) mediopunctatus, Coquillettidia (Rhynchotaenia) chrysonotum, Coquillettidia (Rhynchotaenia) hermanoi, Culex (Culex) sp. pr. usquatus, Culex (Culex) quinquefasciatus, Sabethes (Sabethinus) intermedius, Sabethes (Sabethinus) undosus, Uranotaenia (Uranotaenia) geometrica, Uranotaenia (Uranotaenia) nataliae, Culex (Melanoconion) sp. gr. intricatus, Anopheles (Nyssorhynchus) benarrochi, Anopheles (Nyssorhynchus) rangeli, Anopheles (Anopheles) peryassui, Psorophora (Grabhamia) sp. pr. confinnis, Sabethes (Sabethinus) sp. pr. whitmani, Culex (Melanoconion) sp. pr. educator Psorophora (Janthinosoma) albipes.

A Tabela mostra a lista das espécics cncontradas na área e a quantidade colctada. Obıcve-se um total de 4.588 culicídeos, representando 53 espécies ou grupos.

A espécie mais coletada foi Anopheles (Nyssorhynchus) oswaldoi com 3.156 indivíduos ( 3.133 fêmeas e 23 machos) (68,8\% do total colclado, $86,0 \%$ do total de anofelinos).

Mesmo considerando-se as limitações dessas coletas, o resultado revelou a presença marcante de An. oswaldoi $\mathrm{cm}$ uma região considerada de transmissão de malária pela FNS. Acrescenta-se a isso o encontro de 16 excmplares dessa espécie infectados com Plasmodium vivax e 11 com Plasmodiurn falciparum, detectados em análise preliminar em um love de mil exemplares (das 3.133 fêmcas colctadas por essa equipe) que foram encaminhados para teste de esporozoíto (método: "Enzyme Linked Immunosorbent Assay") ao Instituto de Medicina Tropical da USP (Branquinho ${ }^{3}, 1991$ ).

Torna-se necessário um estudo mais profundo sobre a atividade de anofelinos para que se possa avaliar melhor os vetores cnvolvidos na transmissão da malária na região.

\section{Agradecimentos}

Ao Técnico Francisco Altair de Lima Taveira da Fundação Nacional de Saúde de Rio Branco, Acre, e sua equipe, pclo auxílio durante os trabalhos de campo.

NATAL, D. et al. [Note on the culicidac (Diptera: Culicidac) of the River Purus Basin, Acre, Amazonian, Brazil. Rev. Saúde públ., S. Paulo, 26: 129-31, 1992. Mosquito (Diptera: Culicidae) collections were made on the Pedro Peixolo Colonization Project in the State of Acre, Brazil. Four thousand, five hundred and eightyeight $(4,588)$ specimens were collected and fifty-three (53) species or group recognised. The occurrence of Anopheles (Nyssorhynchus) oswaldoi is given special emphasis.

Keywords: Mosquitoes. Ecology, vectors.

\section{Referências Bibliográficas}

1. ARNELL, J. H. Mosquito studies (Diptera: Culicidae) XXXII - A revision of the scapularis group of Aedes (Ochlerolatus). Contrib. Amer. ent.Inst., 13:1-144,1976.

2. ARRUDA, M. et. al. Potenival vectors of malaria and their different susceptibility to Plasmodium falciparum and Plasmodium vivax in Nonthem Brazil identified by immunoassay. Amer. J. trop. Med. Hyg., 35:873-81, 1986.

3. BRANQUINIIO, M. S. ct. al. Anopheles oswaldoi an important potential vector of malaria in a agricultural project of Acre, Brazil. In: International Congress on Malaria and Babesiosis, 6\%, Rio de Janeiro, Brasil. Abstract. Rio de Janciro, Ministério da Saúde, FIOCRUZ, 1991. p. 10-2.

4. DEANE, L. M. et al . Studies on Brazilian Anophelines from the Northeast and Amazon Regions. Baltimore, The Johns Ilopkins Press., 1946 (The Amcrican Joumal of llygicne. Monographic Series, $\left.n^{2} 18\right)$.

5. FARAN, M. E. Mosquito studies (Diptera: Culicidae) XXXV - A revision of the albimanus scction of the subgenus Nyssorhynchus of Anopheles. Contrib. Amer. ent.Inst., 15:1-215, 1980. 
6. FARAN, M.E. \& LNNTHICUM, K. J. A handbook of the Amazonian species of Anopheles (Nyssorhynchus) (Diptera: Culicidae). Mosquito System., 13:1-81, 1981.

7. FORATTINI, O. P. Enlomologia médica. São Paulo, Ed. USP, $1962 / 1965$. v. 1-3.

8. GALINDO, P. et al. A revision of the Uranotaenia of Panama with notes on other American species of the genus. Ann. ent. Soc. Amer., 47:107-77, 1954

9. GUEDES, A. S. \& MIGUEL, A. de S. Sobre Psorophora (Janthinosoma) albigenu Lutz 1908 e Psorophora (Janthinosoma) albipes (Theobald, 1907) (Diptera: Culicidae). Rev. bras. Malar., 4: 471-86, 1964.

10. LANE, J. Neotropical Culicidae. São Paulo, Ed. USP, 1953. $2 v$.

11. LINTHICUM, K. J. A revision of the Argyritarsis Section of the Nyssorhynchus of Anopheles (Diptera: Culicidae). Mosquito System., 20: 98-271, 1988.
12. ROSA-FREITAS, M.G. Anopheles (Nyssorhynchus) deaneorum: a new species in the albitarsis complex (Diptera: Culicidae). Mem. Inst. Oswaldo Cruz, Rio de Janeiro, 84: 535-43, 1989.

13. SIRIVANAKARNS, $S$. A review of the systematics and a proposed scheme of intemational classification of the new world subgenus Melanoconion of Culex (Diptera: Culicidae). Mosquito System., 14: 265-333, 1982.

14. TADEI, W. P. ct al. Biologia de anofelinos amazônicos. VIII - Conhecimento sobre a distribuição de espécies de Anopheles na região de Tucuruí - Marabá (Pará). Acta amazôn., 13: 103-40, 1983.

Recebido para publicação em 2/12/1991

Reapresentado em 9/3/1992 Aprovado para publicação em 24/3/1992 